\title{
OBTENÇÃO DE DADOS PARA SIMULAÇÃO COMPUTACIONAL COMO FERRAMENTA PARA PREVISÃO DA REAÇÃO DE SÍNTESE DE (Z)-5-BENZILIDENO-2,4- TIAZOLIDINADIONA: USO DO MÉTODO MONTE CARLO PARA ESCOLHA DO SOLVENTE
}

\author{
Y. OLIVEIRA ${ }^{1}$, R. O. SILVA ${ }^{2}$, K. COUTINHO ${ }^{3}$ e M. S. A. PALMA ${ }^{2}$ \\ ${ }^{1}$ Universidade de São Paulo, Instituto de Química \\ ${ }^{2}$ Universidade de São Paulo, Departamento de Tecnologia Bioquímico-Farmacêutica \\ ${ }^{3}$ Universidade de São Paulo, Instituto de Física \\ E-mail para contato: msapalma@usp.br
}

\begin{abstract}
RESUMO - O presente estudo utiliza o método Monte Carlo como ferramenta computacional para a escolha do solvente que otimize o processo de síntese do (Z)-5-benzilideno-2,4-tiazolidinadiona realizado experimentalmente em microrreator capilar. A 2,4-tiazolidinadiona (TZD) e seus derivados constituem um importante grupo farmacofórico no combate de doenças como a diabete tipo melitus e, devido a isso, estudos que promovam a otimização dos processos de síntese tem sido cada vez mais importantes. A utilização do microrreatores de fluxo contínuo já são alternativas que melhoram as condições operacionais, não apenas por aspectos relacionados ao rendimento, mas por serem comprovadamente mais seguros e gerar substancialmente menos resíduos. Discutiremos neste trabalho, em desenvolvimento, verificar a interação de solventes (metanol, etanol e n-propanol) em diferentes temperaturas (78, 98, $120 \mathrm{e}$ $140^{\circ} \mathrm{C}$ ) sobre o rendimento das reações e comparando os parâmetros termodinâmicos ( $E \mathrm{a}, \Delta H, \Delta S$ e $\Delta G)$ obtidos por simulação matemática através do método Monte Carlo. O modelo matemático será ajustado aos dados experimentais de sínteses realizadas no microreator capilar em fluxo contínuo. Foi verificada a influência do solvente e da temperatura no rendimento do produto obtido da reação entre TZD com o benzaldeído. Os resultados preliminares mostram que o melhor solvente para esta reação é o etanol. Para a continuação deste estudo, o modelo matemático será ajustado aos dados experimentais para obter a energia de estabilização dos reagentes e produto e determinar o melhor potencial de interação entre os solutos e o solvente.
\end{abstract}

\section{INTRODUÇÃO}

Os estudos com os derivados da Tiazolidianodiona (TZD) têm recebido cada vez mais incentivos devido a suas propriedades hipoglicemiantes, que reduzem a resistência à insulina sistêmica pelos tecidos periféricos (Jain et al., 2013). Os derivados da TZD tem diversas aplicações na síntese de compostos com atividades farmacológicas e terapêuticas importantes devido a sua diversificada atividade biológica (Mohanty et al., 2015) como anticancerígena, 
anti-HIV, anti-convulsionante, antimicrobiana, anti-histamínica, anti-hipoglicêmica (diabetes melitos), antifúngica, amebicida, anti-inflamatória, entre outros (Lima, 1998; Bahare et al., 2015). A Figura 1 apresenta a estrutura molecular da TZD.

Figura 1 - Estrutura molecular da 2,4-tiazolidinadiona (TZD).

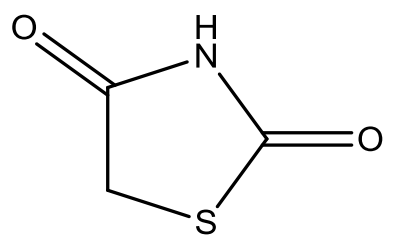

Neste projeto foi sintetizada a (Z)-5-benzilideno-2,4-tiazolidinadiona conforme esquema reacional mostrado na Figura 2 (Luo et al., 2010). Este produto pode ser utilizado como intermediário de molécula com atividade biológica confirmada (Mishra et al., 2015).

Figura 2 - Esquema da reação da 2,4-tiazolidinadiona (A) com o benzaldeído (B) fornecendo o produto (Z)-5-benzilideno-2,4-tiazolidinadiona (C).<smiles>O=C1CSC(=O)N1</smiles>

A<smiles>CC(=O)c1ccccc1</smiles>

B<smiles>Cc1ccc(C=C2SC(=O)N(O)C2=O)cc1</smiles>

Devido a sua aplicabilidade na indústria farmacêutica é de suma importância aprimorar a síntese desses compostos. A utilização de microrreatores nas indústrias químicofarmacêuticas proporciona a redução do impacto gerado pelos processos com substancial redução de resíduos, sendo uma das principais vantagens deste tipo de equipamento, a velocidade com que a difusão dos reagentes ocorre devido à pequena distância entre os microcanais. A Figura 3 mostra uma fotografia do sistema reacional no microrreator capilar (Wiles e Watts, 2012). 
Figura 3 - Equipamento experimental utilizado na síntese do (Z)-5-benzilideno-2,4tiazolidinadiona

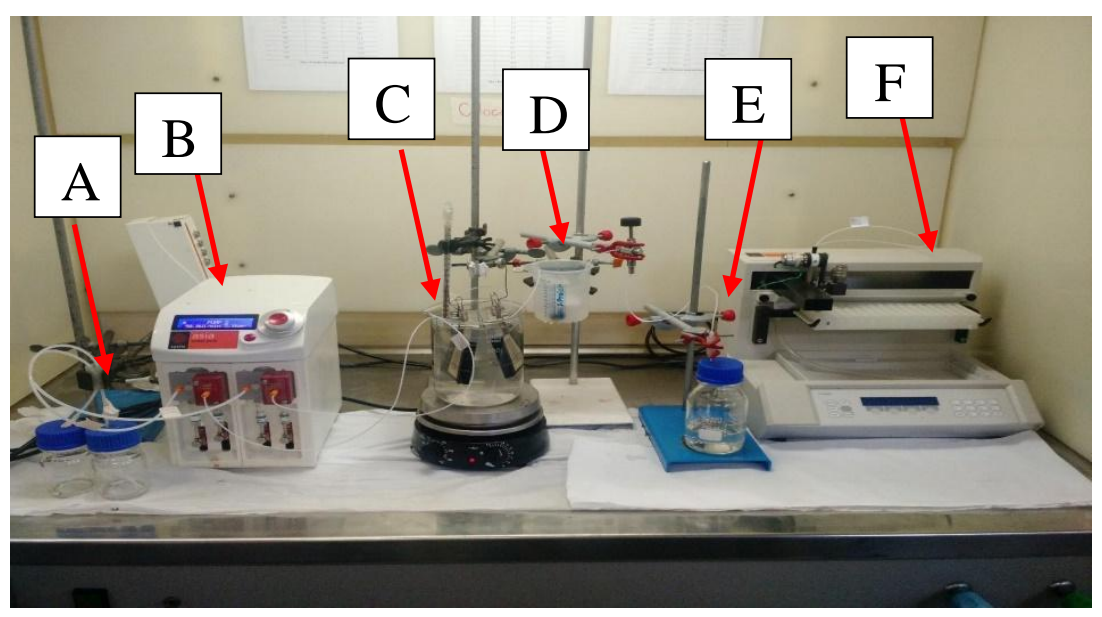

Na Figura 3 estão mostrados os frascos reagentes para a solução de alimentação no microrreator (A), o sistema de bombeamento de dois canais do tipo seringa e pistão (B), os microrreatores de vidro acoplados ao chip header e que estão imersos no sistema de aquecimento (C), o regulador de pressão (D), frasco de coleta de descarte (E) e o sistema de coleta automática de amostras $(\mathrm{F})$.

Neste estudo, como complementação do trabalho experimental usamos o método Sequencial Monte Carlo/Mecânica Quântica, que é um método que tem por objetivo realizar simulações matemáticas considerando o efeito do meio reacional na reação química. Com o método de Monte Carlo é possível obter informações quânticas, através de simulações clássicas da estrutura do líquido, como, por ex., o momento de dipolo do líquido e auxiliar na escolha dos melhores solventes para reações específicas. A simulação computacional permite prever as condições experimentais, em termos de temperatura, pressão e volume, e estimar o comportamento de reações em condições operacionais diferentes e estudar uma reação por um longo período de tempo e com esforço computacional relativamente pequeno (Law, 2007).

O método de Monte Carlo baseia-se em um processo estocástico para gerar configurações moleculares. Neste método, posições atômicas sucessivas são selecionadas aleatoriamente e novas configurações são geradas, de tal forma a satisfazerem a distribuição de probabilidades de Gibbs, o que possibilita obter imagens de configurações acessíveis que fornecem dados relacionados à energia de estabilização das moléculas antes do início da reação de acordo com o solvente escolhido e a estabilidade termodinâmica do produto final (Coutinho, 1998).

\section{MATERIAIS E PROCEDIMENTO EXPERIMENTAL}

\subsection{Materiais}

Na síntese do (Z)-5-benzilideno-2,4-tiazolidinadiona são utilizados TZD, benzaldeído como reagentes; etanol $(99,8 \% \mathrm{~m} / \mathrm{m})$, metanol e n-propanol como solventes no meio reacional, piperidina como catalisador e etanol $(99,5 \% \mathrm{~m} / \mathrm{m})$ no work-up do produto que consiste de 
cristalização e recristalização. Utiliza-se, quando necessário, água destilada.

Na utilização do método de Monte Carlo são geradas as coordenadas cartesianas moleculares obtidas pelo programa Chem 3D Pro®, que são submetidas ao aplicativo computacional de Simulação Sequencial de Método Monte Carlo/Mecânica Quântica desenvolvido no laboratório da Prof ${ }^{a}$. Dra. Kaline Coutinho, do Instituto de Física da Universidade de São Paulo.

\subsection{Procedimento Experimental}

O processo de síntese no microrreator consiste em, primeiramente, preparar duas soluções de alimentação. Para o preparo da primeira solução, solubilizou-se 4 mmol de TZD e 3,2 mmol piperidina em $30 \mathrm{~mL}$ de etanol $99,8 \%$. No preparo da segunda solução, solubilizouse 4 mmol do respectivo aldeído em $30 \mathrm{~mL}$ de etanol 99,8\%. Posteriormente, as duas soluções previamente preparadas foram alimentadas separadamente ao microrreator na vazão e temperatura adequada. Para o processo de síntese foram empregadas as temperaturas de 78, 98,120 e $140^{\circ} \mathrm{C}$, e foram realizadas amostragens para tempos médios de residência (tempo espacial, $\tau$ ) de 20 minutos. O produto sólido obtido nas reações foi posteriormente purificado por cristalização e recristalização com etanol 99,5\%. A quantificação da TZD, do aldeído e do produto formado foi realizada por HPLC-UV com método adaptado de Gumieniczek et al. (2010). O processo foi realizado da mesma forma para metanol e n-propanol sob as mesmas condições.

\subsection{Procedimento Teórico}

O procedimento com método Monte Carlo se divide em duas etapas:

1) Termalização: a partir de uma configuração aleatória de alta energia das moléculas do líquido e através de passos da adotados no método Monte Carlo, leva-se o sistema a uma situação, na qual, a energia oscile entre uma energia de equilíbrio térmico, ou seja, gerar configurações que estejam de acordo com a probabilidade de Gibbs;

2) Simulação: com a configuração do líquido em equilíbrio realiza-se a interação entre solvente e soluto e retira-se as informações estatísticas das propriedades termodinâmicas do sistema.

Realizada a termalização e a simulação se pode calcular as médias das propriedades termodinâmicas do sistema ou, caso estejamos utilizando uma metodologia híbrida, implementar os cálculos quânticos sobre estruturas supramoleculares descorrelacionadas.

\section{RESULTADOS E DISCUSSÕES}

Os resultados experimentais dos rendimentos dos ensaios realizados em microrreator nas temperaturas de $78,98,120$ e $140^{\circ} \mathrm{C}$, para cada solvente, estão apresentados na Tabela 1. 
Tabela 1- Rendimento do produto em diferentes temperaturas com tempo de residência 20 $\min$.

\begin{tabular}{|c|c|c|c|c|}
\hline \multirow{5}{*}{ Solvente } & \multicolumn{5}{|c|}{ Rendimento (\%) } \\
\cline { 2 - 5 } & $\mathbf{7 8}$ & $\mathbf{9 8}$ & $\mathbf{1 2 0}$ & $\mathbf{1 4 0}$ \\
\hline Metanol & 39,1 & 16,7 & 33,6 & 30,9 \\
\hline Etanol & 9,6 & 16,8 & 42,4 & 56,1 \\
\hline n-Propanol & - & 17,2 & 23,4 & 26,0 \\
\hline
\end{tabular}

A Tabela 1 mostra que os maiores valores de rendimento de produto foram obtidos para temperaturas de 120 e $140^{\circ} \mathrm{C}$. Na temperatura de $78^{\circ} \mathrm{C}$ o maior rendimento foi obtido para o metanol (nesta temperatura não foi realizado ensaio para o n-propanol, pois ficaria abaixo da sua temperatura de ebulição e consequentemente o rendimento seria muito baixo. A $98^{\circ} \mathrm{C}$ os resultados de rendimento são muito semelhantes para os 3 solventes, não havendo diferenças significativas. O pior desempenho entre os três solventes foi observado nos ensaios com propanol.

\section{CONCLUSÕES E PERSPECTIVAS}

Nas condições estudadas a temperatura de $140^{\circ} \mathrm{C}$ apresentou os melhores resultados. Com o aumento da temperatura, maior será a interação molecular, velocidade de reação e, consequentemente, aumentará o rendimento do produto obtido. A influência da temperatura foi maior para a reação com o propanol. Pode-se notar a queda do rendimento relacionada ao aumento da temperatura de $78^{\circ}$ para $98^{\circ} \mathrm{C}$. Na próxima etapa do trabalho a reação será estudada computacionalmente através da simulação pelo método de Monte Carlo. Serão obtidas as energias de estabilização das moléculas de reagentes e produto da reação para modelar matematicamente os resultados experimentais. $\mathrm{O}$ trabalho ora em execução tem por objetivo determinar propriedades espectroscópicas de sistemas em fase liquida e determinar o rendimento da reação para outras condições operacionais de temperatura e solventes com baixo esforço computacional. Espera-se que, uma vez obtido o bom ajuste do modelo matemático aos resultados experimentais se possa abrir uma nova frente de pesquisa para investigação do efeito do solvente e temperatura no rendimento de reações químicas de interesse científico e tecnológico.

\section{REFERÊNCIAS}

BAHARE, R.S.; GANGUlY, S.; CHOOWONGKOMON, K.; SEETAHA, S. Synthesis, HIV-1 RT inhibitory, antibacterial, antifungal and binding mode studies of some novel N-substituted 5-benzylidine-2,4-thiazolidinediones. DARU Journal of Pharmaceutical Sciences, v.23, n.6, p.1-15, 2015. 
COUTINHO, K.; OLIVEIRA, M.J.; CANUTO, S. Sampling configurations in Monte Carlo simulations for Quantum mechanical studies of solvent effects. International Journal of Quantum Chemistry, v.66. p.249-253,1998.

GUMIENICZEK, A.; KOMSTA, L. Stability-Indicating Validated HPLC Method for Simultaneous Determination of Oral Antidiabetic Drugs from Thiazolidinedione and Sulfonylurea Groups in Combined Dosage Forms. Journal of AOAC International, v.93, n.4, p. 1086-1092, 2010.

JAIN, V.S.; VORA, D.K.; RAMAA, C.S. Thiazolidine-2,4-diones: Progress towards multifarious applications. Bioorganic \& Medicinal Chemistry, v.21, p.1599-1620, 2013.

LAW, A.M., Simulation Modeling and Analysis (4th ed.), McGraw-Hill, New York, 2007

LIMA, J.G. Alguns Aspectos Químicos do Anel 2,4-tiazolidinadiona. Revista Universidade Rural, Série Ciências Exatas e da Terra, v.20, p.1-8, 1998.

LUO, Y.; MA, L.; ZHENG, H.; CHEN, L.; LI, R.; HE C.; YANG, S.; YE, X.; CHEN, Z.; LI, Z.; GAO, Y.; HAN, J.; HE, G.; YANG, L.; WEI, Y. Discovery of (Z)-5-(4Methoxybenzylidene)thiazolidine-2,4-dione, a Readily Available and Orally. Active Glitazone for the Treatment of Concanavalin A-Induced Acute Liver Injury of BALB/c Mice. Journal of Medicinal Chemistry, v.53, p.273-281. 2010.

MISHRA, G.; SACHAN, N.; CHAWLA, P. Synthesis and Evaluation of ThiazolidinedioneCoumarin Adducts as Antidiabetic, Anti-Inflammatory and Antioxidant Agents. Letters in Organic Chemistry, v.12, p.429-445, 2015.

MOHANTY, S.; ROY, A.K.; KUMAR, V.K.P.; REDDY, S.G.; KARMAKAR, A.C. Acetic anhydride-promoted one-pot condensation of 2,4-thiazolidinedione with bisulfite adducts of aldehydes. Tetrahedron Letters, v.55, p.4585-4589, 2014.

WILES, C.; WATTS, P. Continuous flow reactors: a perspective. Green Chemistry, v.14, p.38-54, 2012. 